\title{
Vascular Infiltration by Hepatocytes
}

National Cancer Institute

\section{Source}

National Cancer Institute. Vascular Infiltration by Hepatocytes. NCI Thesaurus. Code C161539.

Presence of normal hepatocytes in hepatic veins and within the contour of the vessel. (INHAND) 